\title{
Lipid bilayer thickness measured by quantitative DIC reveals phase transitions and effects of substrate hydrophilicity Supplementary Material
}

\author{
David Regan, ${ }^{1}$ Joseph Williams, ${ }^{2}$ Paola Borri, ${ }^{2}$ and Wolfgang Langbein ${ }^{1}$ \\ ${ }^{1}$ School of Physics and Astronomy, Cardiff University, \\ The Parade, Cardiff CF24 3AA, UK* \\ ${ }^{2}$ School of Biosciences, Cardiff University, \\ Museum Avenue, Cardiff CF10 3AX, UK
}

(Dated: October 21, 2019)

\footnotetext{
*ReganDC@cardiff.ac.uk
} 


\section{CONTENTS}

S1. Energy Minimisation of qDIC Phase $\quad 3$

S2. Effect of Defocussing on Phase Measurements 4

S3. Effect of Reproducibility of Polariser Rotation on Phase Measurements 6

S4. Effect of DIC Image Averaging on Phase Measurements 6

S5. Measurement of Glass Noise $\quad 8$

S6. Variation of Bilayer Coverage Across Sample 9

$\begin{array}{ll}\text { S7. Absolute Values of Normalised Data } & 10\end{array}$

S8. Domain Thickness Versus Domain Area 11

S9. Fluorescence and Thickness of Double Bilayer Domains 12

S10. Optical Thickness Difference Between $\mathrm{L}_{\mathrm{o}}$ and $\mathrm{L}_{\mathrm{d}}$ Domains 14

$\begin{array}{ll}\text { References } & 15\end{array}$ 

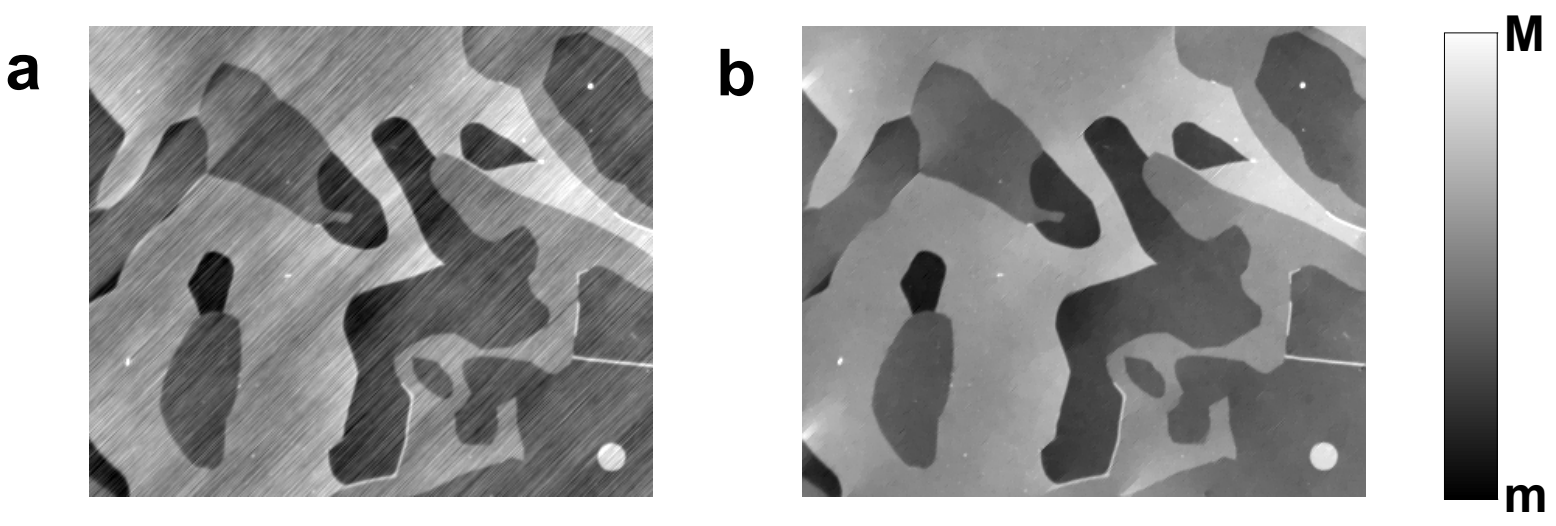

FIG. S1. An example of a qDIC phase image showing a single bilayer patch in a $(39 \times 33) \mu^{2}$ region a) using Wiener deconvolution only, and b) after energy minimisation, scaled from -10 to 10 mrad. The Wiener deconvolution used a signal to noise ratio of 4000, and the energy minimisation used a smoothness power of 0.5 and factor of $10^{-6}$.

\begin{tabular}{|l|l|l|l|}
\hline Figure & Smoothness power $\alpha$ & Smoothness weight $\lambda$ & $\#$ Iterations \\
\hline Fig. 1 & 0.5 & $1 \times 10^{-6}$ & 2001 \\
Fig. 6 & 0.5 & $1 \times 10^{-6}$ & 27001 \\
\hline
\end{tabular}

TABLE SI. Parameters used in the qDIC global minimisation for the different figures shown in the main text

\section{S1. ENERGY MINIMISATION OF QDIC PHASE}

qDIC phase images resulting from Wiener deconvolution [1] tend to suffer from stripe-like artefacts running parallel to the shear direction, as can be seen in Fig. S1a. They are the results of the fact that only the phase gradient in one direction is measured, and not the phase itself, and thus information about the gradient orthogonal to the DIC shear direction is missing. For the analysis of bilayer steps, this is not problematic, as it is sufficient to take phase profiles close to parallel to the shear, and fitting with the function Eq.(1) of the main paper which includes a linear slope to accommodate the long-range artefacts resulting from the integration. However, the stripes affect the visibility of low contrast objects such as $\mathrm{L}_{\mathrm{o}}$ domains in the qDIC phase images.

These artefacts can be reduced using a global minimisation method, varying the qDIC phase image to minimise not purely the deviation between measured and simulated DIC 


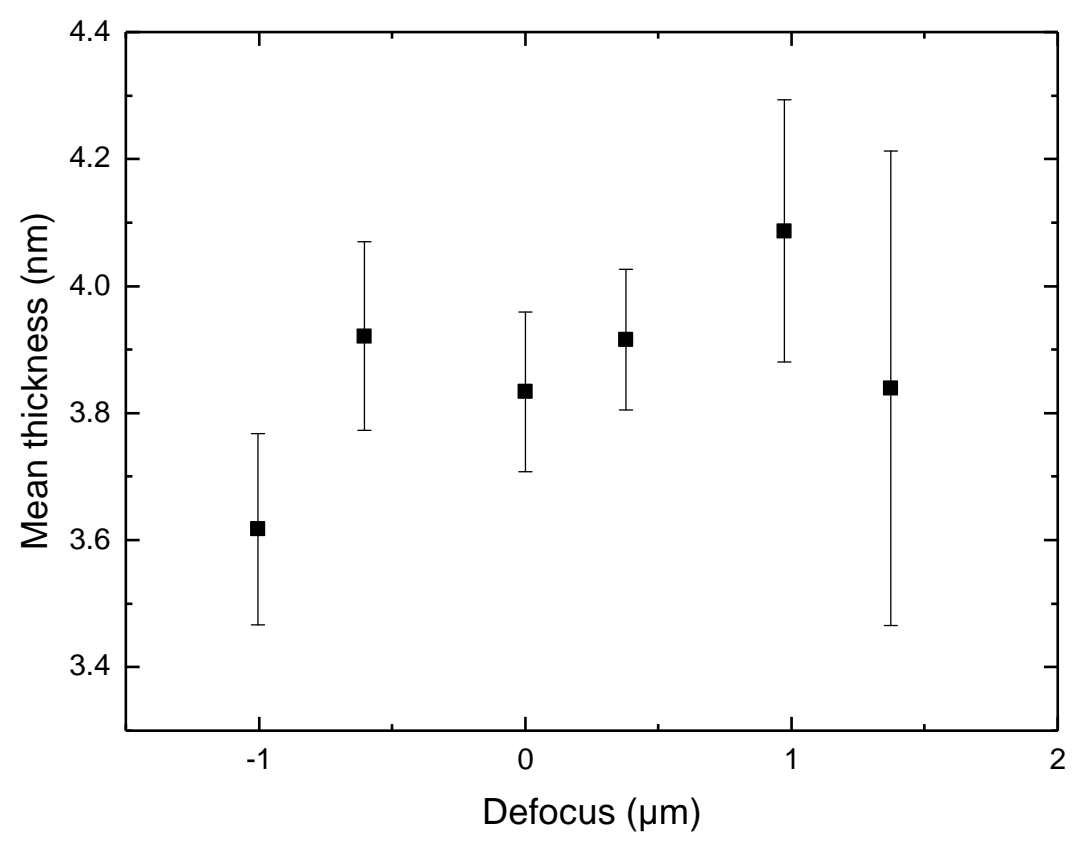

FIG. S2. Thickness measurements for a given region of interest taken with the objective at different heights relative to the position of best focus. As the sample is mounted on an inverted microscope, increasing objective height corresponds to reduced separation from the sample. The error bars represent the standard error.

contrast, but the sum of this deviation, and the magnitude of the gradient, elevated to a power $\alpha$ and multiplied with a weight $\lambda$. For a power between zero and one, small gradients carry a higher penalty, resulting in qDIC phase images with flat regions connected by steps, consistent with the bilayer structure. The power and the weight have to be chosen suitably to provide a small minimum step height while still flattening regions dominated by measurement noise. This method, inspired by Koos et al. [2], and described in detail in [3], is implemented in MATLAB R2015a, and for clarity most images in the main body of the paper have been processed in this way. Table SI gives the parameters used for the various images shown in the present work.

\section{S2. EFFECT OF DEFOCUSSING ON PHASE MEASUREMENTS}

After focussing the DIC image at the sample centre, the focus often varied across the image by up to $\pm 1 \mu \mathrm{m}$ due to residual sample tilt. Furthermore, during measurements, 


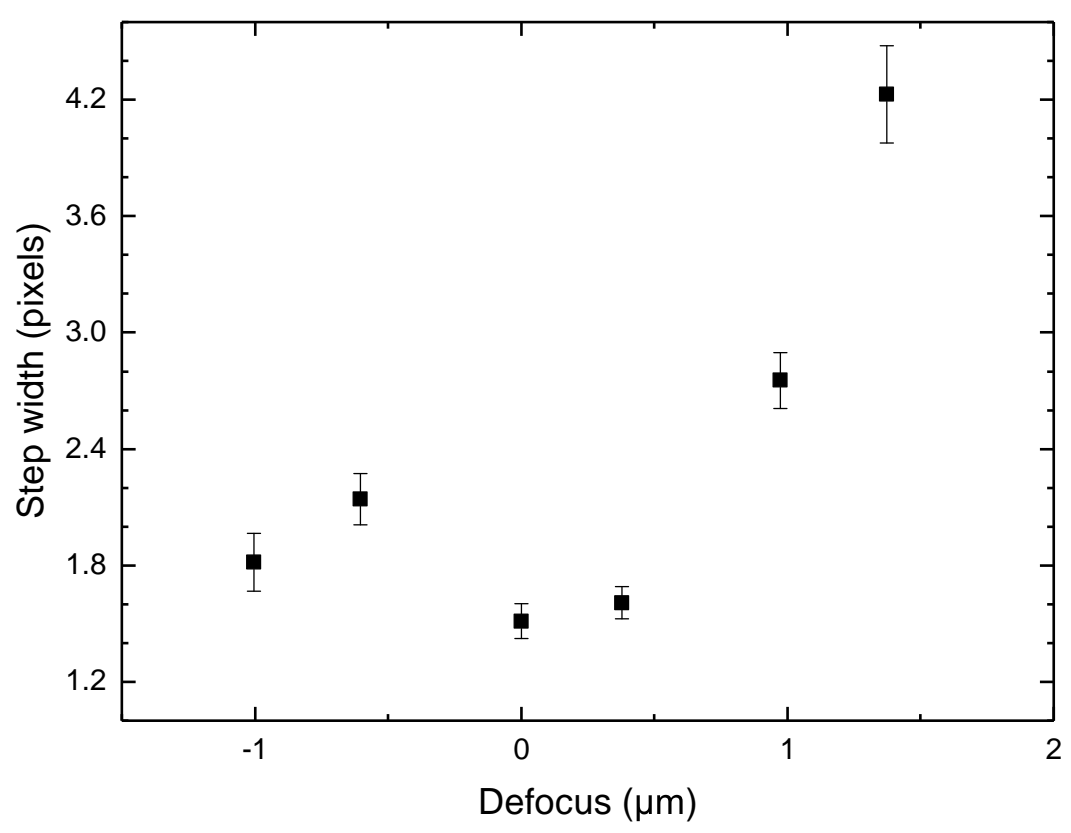

FIG. S3. The width $c$ of function Eq.(1) fitted to the data as a function of defocus. The error bars represent the standard error.

focal drift of a similar scale was observed due to, for example, thermal drift between image acquisition. To determine the effect of this undesired defocus on the retrieved step heights, the same region of interest was imaged multiple times at different defocus. A total of ten line profiles were taken in each image, with the positions of the line profiles kept constant between images. The number of profiles used is much smaller than used for the mean thicknesses reported in the manuscript, but is sufficient to observe significant systematic changes with defocus.

The resulting mean thickness as function of defocus is shown in Fig. S2. The error bars for the mean values at different degrees of defocus overlap, indicating that the mean bilayer thickness was not significantly affected over the range of axial positions studied. It can also be seen that the changes in mean thickness with defocus are effectively random, so defocus is not expected to bias the mean values towards higher or lower thicknesses. A small degree of focal drift across the field of view was therefore deemed permissible. It should be pointed out that the extremes of defocus explored here are easily visible in the images, and in practise the sample would have been refocussed before taking data. The width of the function fitted to the data (the $c$ parameter in Eq.(1)) was increasing with defocus (see Fig. S3), as expected. 


\section{S3. EFFECT OF REPRODUCIBILITY OF POLARISER ROTATION ON PHASE MEASUREMENTS}

For many measurements, the polariser had to be calibrated manually. This was done by adjusting the polariser until the mean number of counts detected by the camera matched the expected value calculated from Eq.(S1).

$$
N(\theta)=\left(N_{0}-N_{\mathrm{BG}}\right) \sin ^{2}(\theta)+N_{\mathrm{BG}}
$$

In Eq. (S1), $N_{0}$ is the number of counts detected when the polariser angle is $90^{\circ}$, corresponding to an excitation polarisation parallel to the DIC analyser, ensuring that the camera

was not being saturated. $N_{\mathrm{BG}}$ is the average number of dark counts of the camera, which is measured by taking the average number of counts when the light from the objective is directed away from the camera to the eyepiece. The same region was imaged three times at a nominal polariser angle of $12.9^{\circ}$ with the calibration of the polariser redone each time. Multiple line profiles were taken at the same positions in the three images. The resulting average thicknesses were $(3.75 \pm 0.04) \mathrm{nm},(3.72 \pm 0.05) \mathrm{nm}$ and $(3.77 \pm 0.05) \mathrm{nm}$, equal within the experimental precision.

\section{S4. EFFECT OF DIC IMAGE AVERAGING ON PHASE MEASUREMENTS}

Due to the small levels of DIC contrast generated by lipid bilayers, individual frames result in a noise only a few times below the bilayer step signal. To reduce the noise, the DIC images were averaged over $N$ frames taken at each polariser orientation. Increasing $N$ reduces the random noise in the images, while also correspondingly increasing the variations of the sample during the measurement time, e.g. due to drift, or bilayer motion.

It was therefore important to determine an appropriate number of frame averages to take during imaging. To this end, a single region of interest was imaged and 1000 individual frames were taken over about 100 seconds, first for positive and then for negative phase offset. The qDIC phase was then determined using $I_{+}$and $I_{-}$averaged over subsets of 1 , 10, 100 and 1000 frames, resulting in the images shown in Fig. S4. A reduction of the image noise with increasing $N$ is observed.

In order to determine the resulting effect on the retrieved bilayer thickness, the qDIC 
a

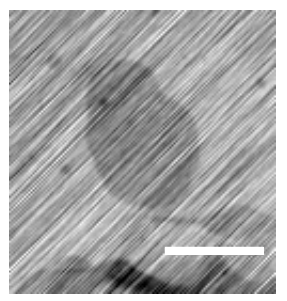

b

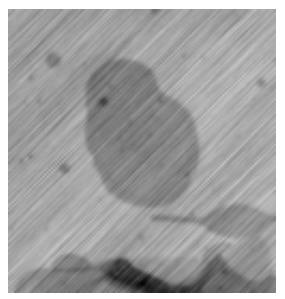

C

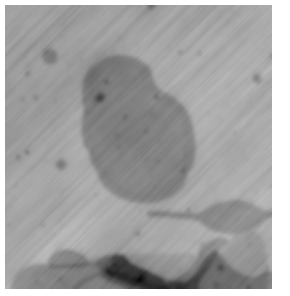

d

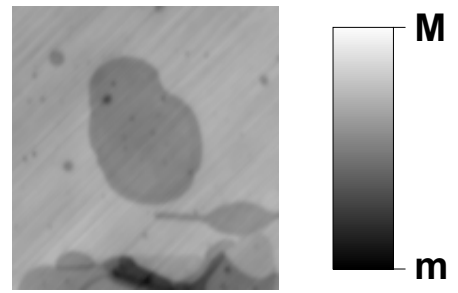

FIG. S4. qDIC phase for a) 1, b) 10, c) 100, and d) 1000 averaged frames. The scale bar represents $10 \mu \mathrm{m}$, and the image contrast ranges from $m=-30 \mathrm{mrad}$ to $M=40 \mathrm{mrad}$.

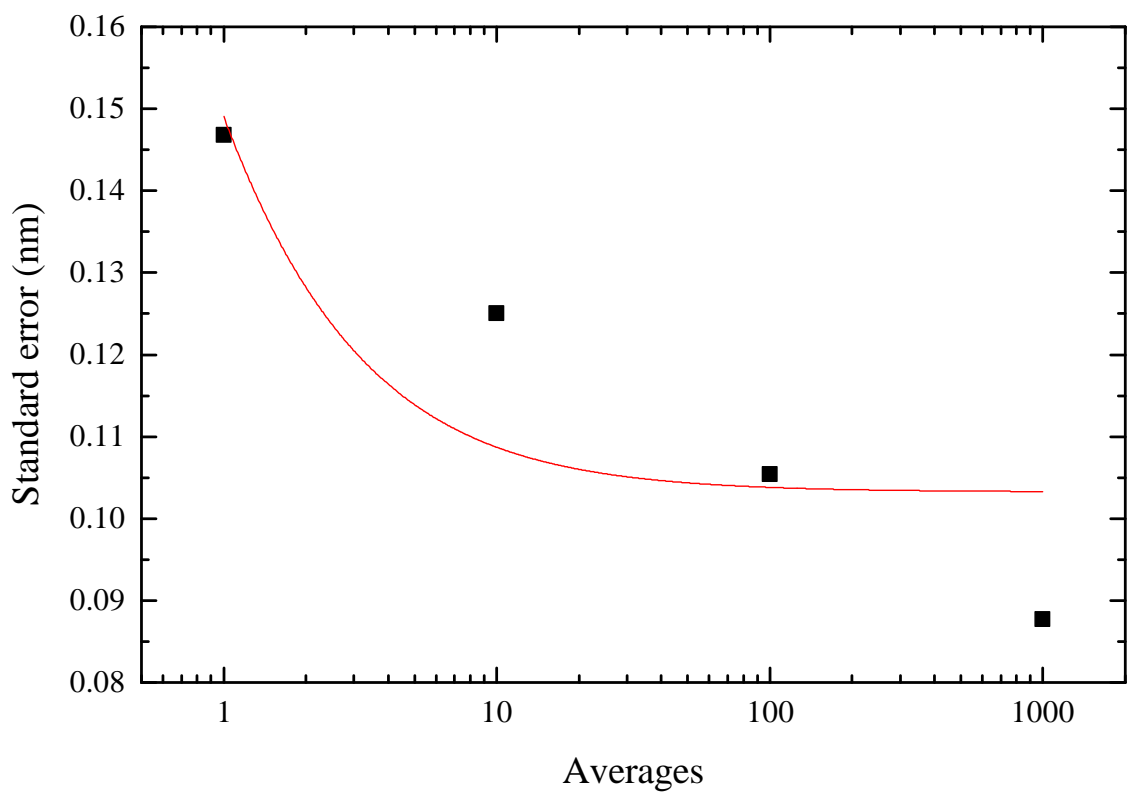

FIG. S5. The effect of number of averages on the standard error of the distribution of single bilayer thickness measurements. The red line is the fit of the expected noise function discussed in text.

phase for the different number of averages was fitted as previously described, again averaging over a line width of 8 pixels. The mean bilayer thickness (averaged over 40 individual steps across four images for each number of averages tested) was the same, within error, for all four noise levels. The standard error of the bilayer thickness decreased slightly with the number of averages, as shown in Fig. S5. We have fitted the data with the expected dependence $\sigma=\sqrt{\sigma_{\mathrm{S}}^{2} / N+\sigma_{\mathrm{G}}^{2}}$, considering the shot-noise component $\sigma_{\mathrm{S}}$ for a single frame, the number of frames $N$, and the glass background component $\sigma_{\mathrm{G}}$, as shown in Fig. S5, resulting in $\sigma_{\mathrm{G}}=$ $0.103 \pm 0.010$ and $\sigma_{\mathrm{S}}=0.107 \pm 0.025$. We note that the fit does not describe the data well, possibly due to the influence of drifts during the measurement period. Based on these results, $N=100$ was chosen for the data shown in the main text, as compromise between 
acquisition speed and noise level.

\section{S5. MEASUREMENT OF GLASS NOISE}

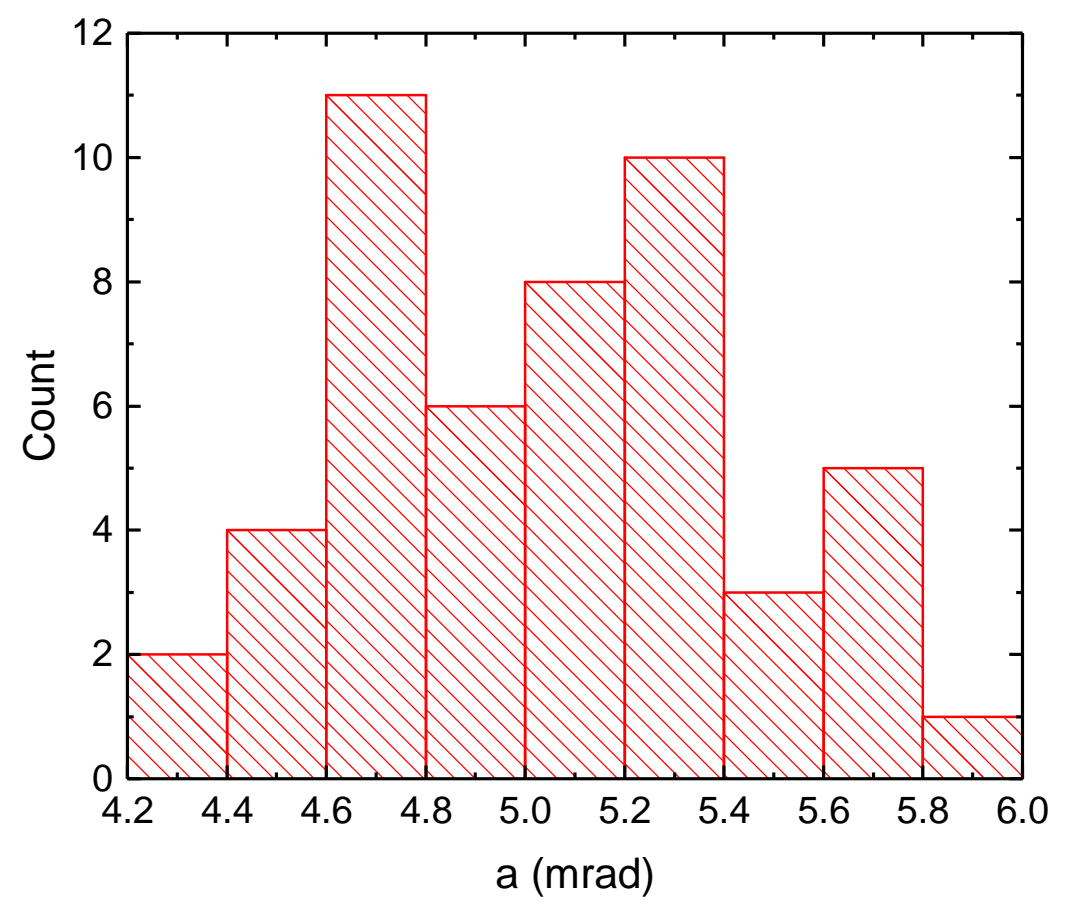

FIG. S6. Histogram showing the distributions of fits to model steps with real glass noise added. The standard deviation of the distribution is $0.407 \mathrm{mrad}$. The "true" value of $a$ is 5.0.

In order to evaluate the noise on the step height due to the surface roughness of the glass, the phase profiles (with a line width of 8 pixels) over 50 random sections of bare glass surface were measured and each added to a mock step function, to simulate the glass noise on an otherwise clean bilayer measurement. The values used to generate the step are based on values typically found fitting to actual single bilayer data. The DIC images of the glass used to generate the phase image from which the line profiles were taken were averaged over 100 acquisitions as normal. These steps were then fitted using the same automated fitting routine as used for measuring bilayer phase steps. The parameters used to generate the mock step and the mean values generated by the fitting routine to the 50 sets of noisy data are shown in table SII. The errors given are the standard error of the mean.

The mean values measured by the fitting procedure are, with the exception of the back- 


\begin{tabular}{l|l|l} 
Parameter & Set value & Mean value \\
\hline $\mathrm{a}$ (mrad) & 5 & $5.02 \pm 0.06$ \\
$\mathrm{~b}$ (pixels) & 15 & $14.97 \pm 0.03$ \\
$\mathrm{c}($ pixels $)$ & 1.65 & $1.68 \pm 0.03$ \\
$\mathrm{~d}(\mathrm{mrad} /$ pixel $)$ & 0 & $(1.84 \pm 0.20) \times 10^{-2}$ \\
e (mrad) & 80 & $79.78 \pm 0.17$ \\
\hline
\end{tabular}

TABLE SII. Effect of glass-roughness on fit parameters.

ground gradient parameter, $d$, equal to the set values within the error. When converted to a thickness value, the error on the phase step value $a$ becomes $0.045 \mathrm{~nm}$. This is of a comparable scale to the experimentally measured errors, indicating that glass noise accounts for most of the observed distribution in thickness values. The slightly larger size of the error in the mock data compared to the real experimental data is likely due to the fact that when taking steps from real data, phase profiles which deviate significantly from the expected step-like shape are excluded, thus the most noisy data is filtered out manually before analysis.

To quantify the roughness of the glass, the spatial standard deviation of the glass surface measurements was taken, without the addition of the mock step function. To exclude the effects of local gradients which are accounted for in our fit function (and thus wouldn't affect our measurements), a linear fit was made to each line profile, and the gradient and offset subtracted before taking the standard deviation. The standard deviation of all our glass phase measurements (a total of 21,424 points) was $0.204 \pm 0.002 \mathrm{mrad}$. This is equivalent to a thickness variation of $0.161 \pm 0.002 \mathrm{~nm}$ using the DOPC refractive index of 1.445 , or $0.097 \pm 0.001 \mathrm{~nm}$ using the glass refractive index of 1.5171 .

\section{S6. VARIATION OF BILAYER COVERAGE ACROSS SAMPLE}

In spin-coated SLBs, the degree of bilayer coverage can vary considerably between different fields of view. Examples are shown in Fig. S7. The degree of coverage is quantified either by using the threshold tool in ImageJ on the fluorescence images to set all pixels covered by lipid to 1 and all uncovered pixels to 0, and measuring then mean image intensity, or by using the ImageJ polygonal selection tool for manual area measurements.

In the case of the bilayer patches, the local coverage is very low, with usually only a 

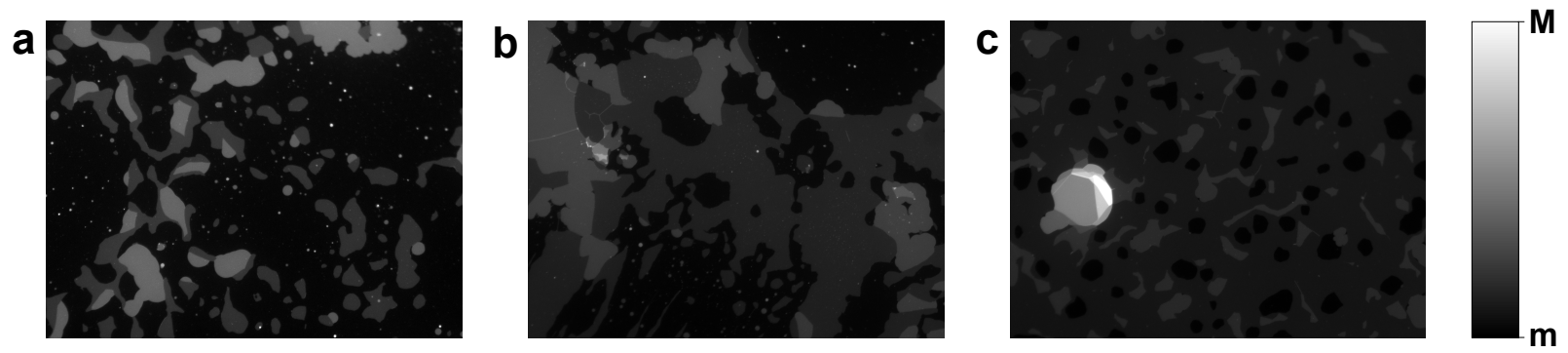

FIG. S7. Fluorescence images of an H-DOPE labelled DOPC SLB with a) $27.5 \%$ coverage $(m=$ 300 pe to $M=1000$ pe), b) $58.2 \%$ coverage $(m=300$ pe to $M=1000$ pe), and c) $88.1 \%$ coverage ( $m=350$ pe to $M=2200 \mathrm{pe}$ ). The images show the full field of view acquired by the camera, an area of $(290 \times 221){\mu \mathrm{m}^{2}}^{2}$.
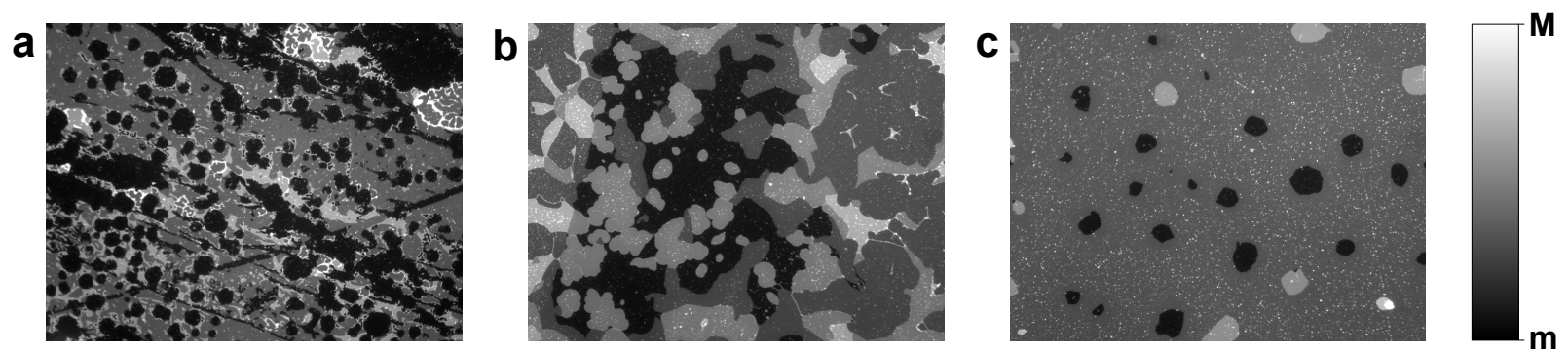

FIG. S8. Fluorescence images of a T-PUPC labelled DOPC SLB with a) $55.0 \%$ coverage $(m=$ 760 pe to $M=2200$ pe), b) $78.4 \%$ coverage $(m=300$ pe to $M=600$ pe), and c) $95.9 \%$ coverage ( $m=350$ pe to $M=2200$ pe). The images show the full field of view acquired by the camera, an area of $(290 \times 221) \mu^{2}$.

couple of bilayer patches visible within a single field of view. The bilayer patches have sizes typically on the order of $10-20 \mu \mathrm{m}$, as shown in Fig. S9. While in some cases the patches may be linked by small stretches of lipid, generally they are unconnected.

\section{S7. ABSOLUTE VALUES OF NORMALISED DATA}

The average bilayer thicknesses presented in normalised form the in Table 1 in the main text are presented in Table SIII as the absolute thickness values. Also included are the raw measurements for the sample formed on the glass treated with hydrogen peroxide rather than piranha etching, which is identified by its hydrophilicity of $4.9^{\circ}$. The number of measurements, $n$, for each value is given in brackets. Errors given are the standard errors of the 
a

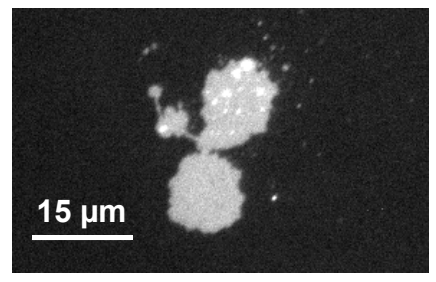

b

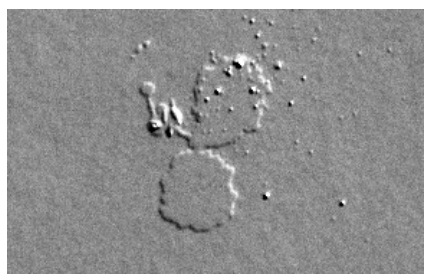

C

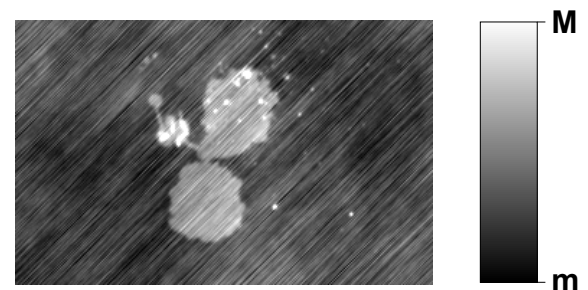

FIG. S9. Images of a DOPC/H-DOPE (99.9/0.1) lipid bilayer patch, scaled from $m$ to $M$, shown in a) fluorescence ( $m=0$ pe to $M=150 \mathrm{pe})$, b) qDIC contrast $(m=-0.00250$ to $M=0.00206)$, and c) qDIC phase $(m=-8.79 \mathrm{mrad}$ to $M=3.40 \mathrm{mrad})$.

mean.

\begin{tabular}{l|l|l|l|l|l}
$\mathrm{CA}\left({ }^{\circ}\right)$ & Medium & Stock & First bilayer $(\mathrm{nm})$ & Second bilayer $(\mathrm{nm})$ & Third bilayer $(\mathrm{nm})$ \\
\hline 3.5 & PBS & Old & $3.992 \pm 0.052(n=65)$ & $4.497 \pm 0.085(n=45)$ & N/A \\
3.5 & $D W$ & Old & $4.826 \pm 0.061(n=83)$ & $5.415 \pm 0.158(n=27)$ & $N / A$ \\
11.3 & PBS & Old & $3.710 \pm 0.054(n=44)$ & $4.000 \pm 0.030(n=106)$ & $4.043 \pm 0.062(n=35)$ \\
3.5 & PBS & Old & $3.801 \pm 0.021(n=186)$ & $4.134 \pm 0.024(n=186)$ & $4.063 \pm 0.102(n=27)$ \\
4.9 & PBS & New & $4.198 \pm 0.022(n=234)$ & $4.682 \pm 0.026(n=342)$ & $4.577 \pm 0.118(n=10)$ \\
41.7 & PBS & New & $4.650 \pm 0.012(n=778)$ & $4.643 \pm 0.019(n=518)$ & $4.524 \pm 0.064(n=56)$ \\
\hline
\end{tabular}

TABLE SIII. Raw thickness measurements not explicitly stated in text, for bilayers formed on surfaces with different contact angles (CAs) and in either PBS or distilled water (DW). Values corresponding to the ratios given in Table 1 are marked in italics. Entries are ordered from oldest to newest date of data acquisition.

\section{S8. DOMAIN THICKNESS VERSUS DOMAIN AREA}

As part of our discussion of the $\mathrm{L}_{\mathrm{o}}$ domain thickness in the main text, it is mentioned that we find no evidence of significant thickness differences between domains. The distribution we observe for both labelled and unlabelled samples is shown in Fig. S10. Additionally, we find no correlation between domain area and thickness. We present the data in Fig. S11 for both labelled and unlabelled samples. It can be seen that there is no clear relationship between area and thickness, within the measurement error. 


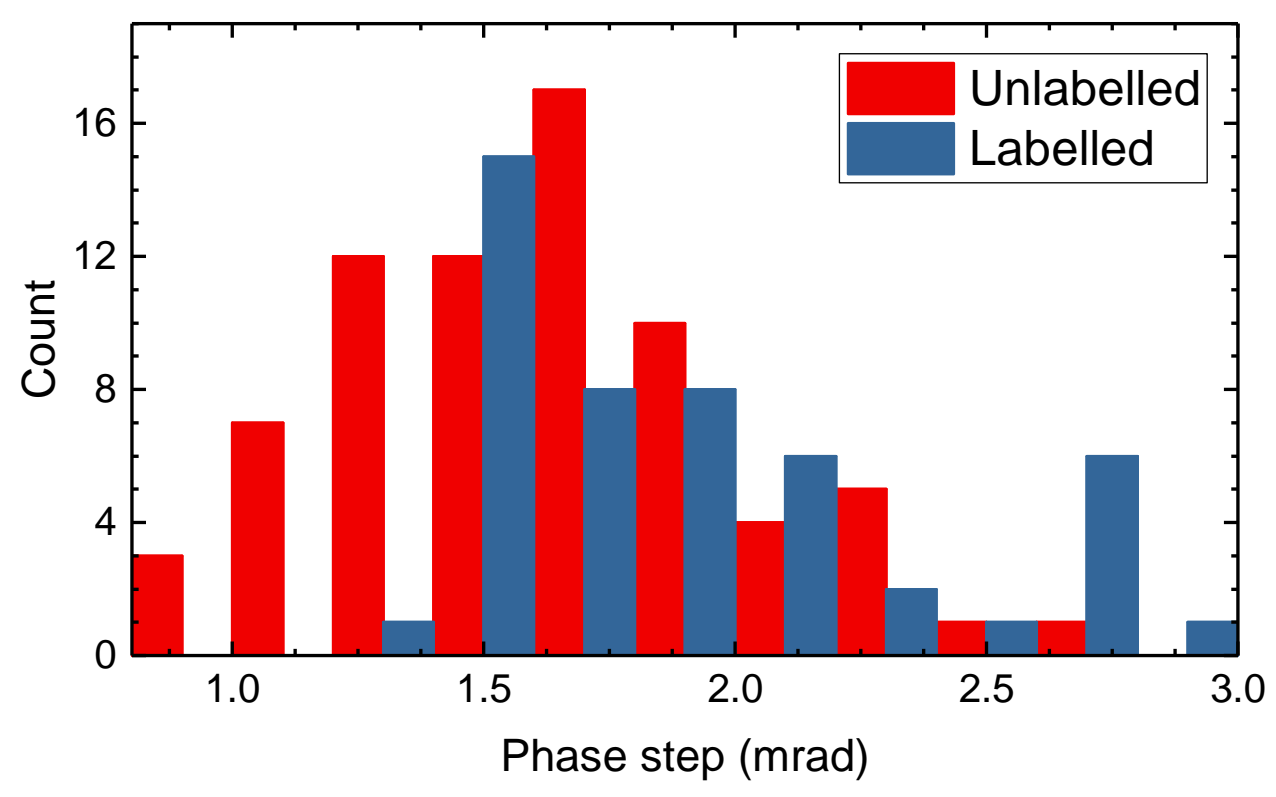

FIG. S10. Plot of the distribution of the phase steps, measured at the boundary between $\mathrm{L}_{\mathrm{o}}$ and $\mathrm{L}_{\mathrm{d}}$ phases, in both labelled and unlabelled samples.

\section{S9. FLUORESCENCE AND THICKNESS OF DOUBLE BILAYER DOMAINS}

As in the DOPC/H-DOPE (99.9/0.1) samples, double bilayer regions can be seen in SLBs formed from the DOPC/SM/Chol/H-DOPE (54.9/25.0/20.0/0.1) mixture. Understanding the phase behaviour in these double bilayer regions can be difficult, but the qDIC technique can give more insight into these regions than fluorescence alone.

For example, in Fig. S12 a single bilayer region can be seen on the right, with a network of tubes on the left. The structure of the bilayer patches that are connected to the tubular network is unclear from the fluorescence alone, however when the qDIC phase image is included as an overlay, the different regions can be clearly identified by their colour. Regions that are a single $\mathrm{L}_{\mathrm{d}}$ phase bilayer appear dark green, while dark red indicates the singlebilayer $\mathrm{L}_{\mathrm{o}}$ phase. Bright red indicates two stacked $\mathrm{L}_{\mathrm{o}}$ bilayers, while orange represents a combination of one $\mathrm{L}_{\mathrm{o}}$ phase bilayer and one $\mathrm{L}_{\mathrm{d}}$ phase bilayer stacked one on top of the other. Notably, using only fluorescence it is not possible to distinguish between double $\mathrm{L}_{\mathrm{o}}$ phase bilayers surrounded by tubes, and regions where the tubes encircle an empty area. The qDIC technique therefore eliminates the need for a second label for the $\mathrm{L}_{\mathrm{o}}$ phase that is otherwise required to accurately interpret the data. 


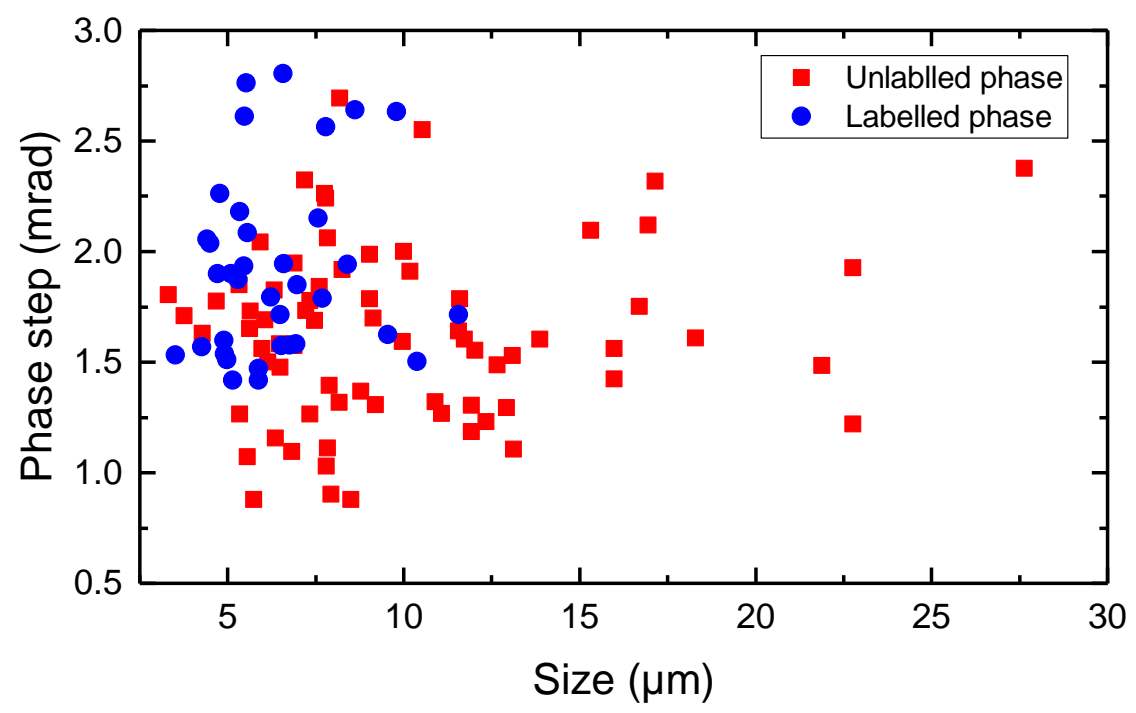

FIG. S11. Optical thickness change over the $\mathrm{L}_{\mathrm{d}}$ to $\mathrm{L}_{\mathrm{o}}$ domain boundary as a function of $\mathrm{L}_{\mathrm{o}}$ domain size, defined as the square root of the domain area. Data from labelled (blue dots) and unlabelled (red squares) samples.
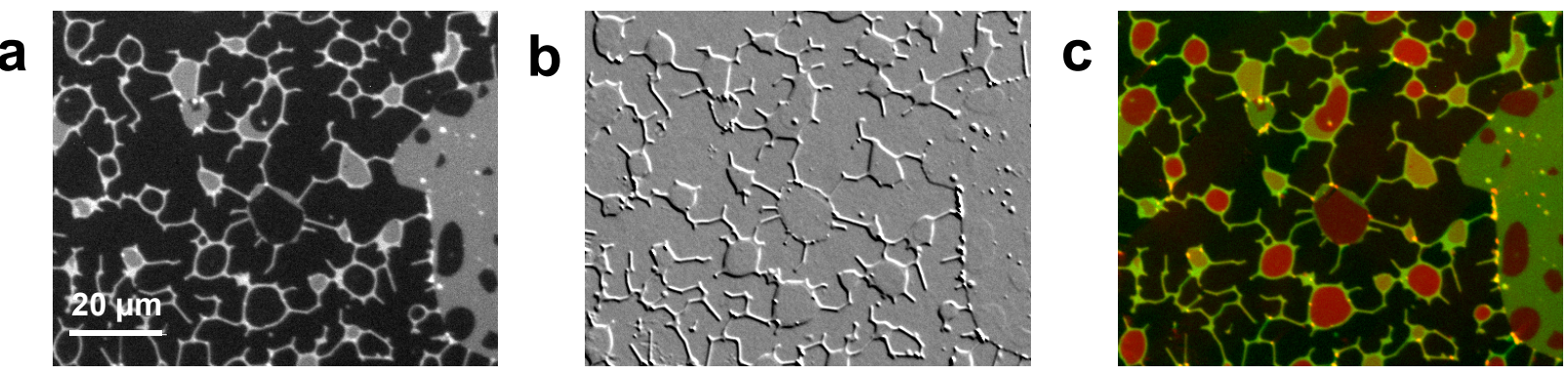

FIG. S12. Region of a DOPC/SM/Chol/H-DOPE (54.9/25.0/20.0/0.1) sample showing a) fluorescence (scaled from 230 to 350 pe), b) qDIC contrast (scaled from -0.00320 to 0.00282), and c) a composite image generated from fluorescence (green) and qDIC phase (red) images.

Different combinations of phases can be differentiated by their relative fluorescence and optical thickness, as can be seen in Fig. S13, where the measured fluorescence and optical thickness values are normalised to the $\mathrm{L}_{\mathrm{d}}$ phase of the first bilayer. The $\mathrm{L}_{\mathrm{d}}+\mathrm{L}_{\mathrm{d}}$ regions have approximately double the fluorescent intensity of the $\mathrm{L}_{\mathrm{o}}+\mathrm{L}_{\mathrm{d}}$ regions, while having approximately the same normalised optical thickness. 


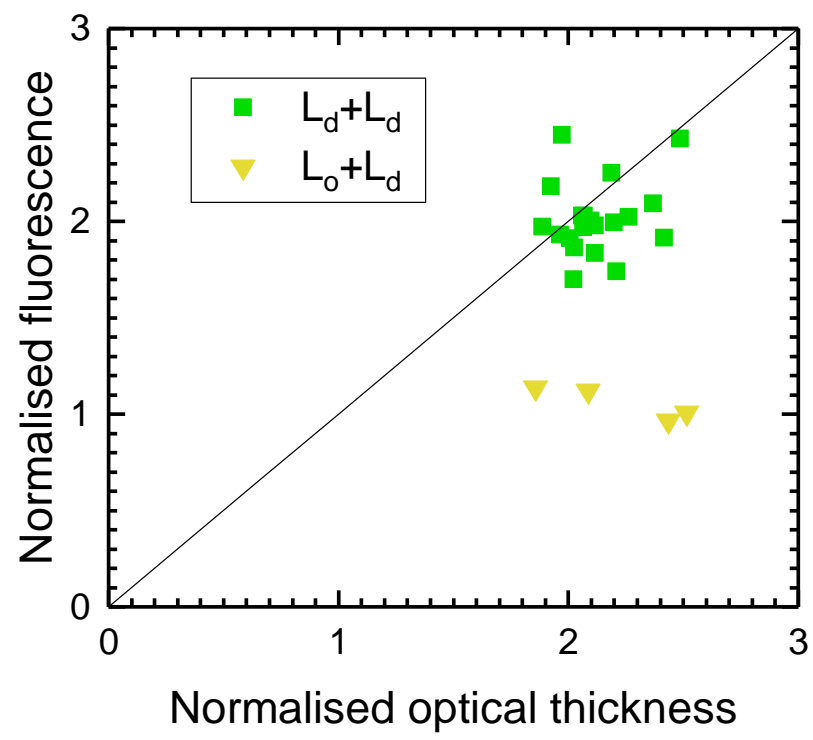

FIG. S13. Fluorescence versus phase for double bilayers in a ternary sample, normalised to single $\mathrm{L}_{\mathrm{d}}$ phase bilayers of the same region. Double $\mathrm{L}_{\mathrm{d}}$ bilayers (green squares) are distinguished from $\mathrm{L}_{\mathrm{d}}+\mathrm{L}_{\mathrm{o}}$ phase bilayers (yellow triangles).

\section{S10. OPTICAL THICKNESS DIFFERENCE BETWEEN L $\mathrm{L}_{\mathrm{O}}$ AND $\mathrm{L}_{\mathrm{D}}$ DOMAINS}

The thicknesses of the $\mathrm{L}_{\mathrm{o}}$ and $\mathrm{L}_{\mathrm{d}}$ domains is dependent on a multitude of factors as discussed in the main body of the paper. These include proximity to the support, the hydrophilicity of the support, and fluorescent labelling. The optical thickness difference between the $\mathrm{L}_{\mathrm{o}}$ and $\mathrm{L}_{\mathrm{d}}$ domains measured for the different conditions are shown in Table SIV.

It is important to note that while we attribute the reduced optical thickness difference in the unlabelled samples compared to the labelled samples to a change of interaction with the substrate, the optical thickness difference in the first bilayer without labelling remains affected by the substrate interaction (see Table 2), and thus can be different to the one in the second bilayer. We find for example that reducing hydrophilicity increases both the $\mathrm{L}_{\mathrm{d}}$ and $\mathrm{L}_{\mathrm{o}}$ phase thickness in the first bilayer, as shown in Fig. 7. However, since the $\mathrm{L}_{\mathrm{o}}$ phase excludes the fluorophore, only the $\mathrm{L}_{\mathrm{d}}$ phase thickness is affected by the presence of the fluorophore. Notably, in the second bilayer the thickness difference is unaffected by labelling, showing that the effect of the labelling on the thickness is due to the interaction 


\begin{tabular}{|c|c|c|c|c|}
\hline Stock & $\mathrm{CA}\left({ }^{\circ}\right)$ & Bilayer & Labelled & Unlabelled \\
\hline Old & 3.5 & First & $\begin{array}{l}1.776 \pm 0.036 \mathrm{mrad}(n=74) \\
1.176 \pm 0.028 \mathrm{~nm}\end{array}$ & $\mathrm{~N} / \mathrm{A}$ \\
\hline Old & 3.5 & First & $\mathrm{N} / \mathrm{A}$ & $\begin{array}{l}1.596 \pm 0.038 \operatorname{mrad}(n=72) \\
1.034 \pm 0.030 \mathrm{~nm}\end{array}$ \\
\hline Old & 3.5 & First & $\begin{array}{l}1.915 \pm 0.059 \mathrm{mrad}(n=48) \\
1.286 \pm 0.047 \mathrm{~nm}\end{array}$ & $\begin{array}{l}1.628 \pm 0.046 \mathrm{mrad}(n=72) \\
1.060 \pm 0.036 \mathrm{~nm}\end{array}$ \\
\hline Old & 11.3 & First & $\begin{array}{l}1.994 \pm 0.048 \operatorname{mrad}(n=91) \\
1.348 \pm 0.038 \mathrm{~nm}\end{array}$ & $\mathrm{~N} / \mathrm{A}$ \\
\hline New & 3.5 & Second & $\begin{array}{l}1.991 \pm 0.027 \mathrm{mrad}(n=290) \\
1.346 \pm 0.021 \mathrm{~nm}\end{array}$ & $\begin{array}{l}1.990 \pm 0.027 \mathrm{mrad}(n=304) \\
1.345 \pm 0.021 \mathrm{~nm}\end{array}$ \\
\hline
\end{tabular}

TABLE SIV. Measured differences in optical thickness between $\mathrm{L}_{\mathrm{o}}$ and $\mathrm{L}_{\mathrm{d}}$ domains for different bilayers, surface contact angles (CA), and lipid stocks. Each row represents a separate measurement session. For context, the corresponding absolute thickness differences are given in italics, calculated assuming a fixed $\mathrm{L}_{\mathrm{o}}$ domain thickness of $5 \mathrm{~nm}$, and the refractive indexes of the two phases given in the main text.

with the substrate.

[1] Iestyn Pope, Lukas Payne, George Zoriniants, Evan Thomas, Oliver Williams, Peter Watson, Wolfgang Langbein, and Paola Borri. Coherent anti-Stokes Raman scattering microscopy of single nanodiamonds. Nat. Nanotechnol., 9:940-, October 2014.

[2] Krisztian Koos, József Molnár, Lóránd Kelemen, Gábor Tamás, and Peter Horvath. DIC image reconstruction using an energy minimization framework to visualize optical path length distribution. Sci. Rep., 6:30420, July 2016.

[3] J. B. Williams. High sensitivity quantitative analysis of differential interference contrast. in Preparation, 2019. 